\title{
A note on the Hausdorff dimension of general sums of pulses graphs
}

\section{Enrique de Amo • Imen Bhouri • Juan Fernández-Sánchez}

Received: 28 February 2011 / Accepted: 8 March 2011 / Published online: 15 July 2011

(C) The Author(s) 2011. This article is published with open access at Springerlink.com

\begin{abstract}
In this work we study the some general fractal sums of pulses defined in $\mathbb{R}$ by:

$$
F(t)=\sum_{n=1}^{+\infty} a_{n} G\left(\lambda_{n}^{-1}\left(t-X_{n}\right)\right)
$$

where $\left(a_{n}\right),\left(\lambda_{n}\right)$ two positive scalar sequences such that $\sum a_{n}$ is divergent, and $\left(\lambda_{n}\right)$ is non-increasing to $0, G$ is an elementary bump and $X_{n}$ are independent random variables uniformly distributed on a sufficiently large domain $\Omega$. We investigate the Hausdorff dimension of the graph of $G$ and in particular we answer a question given by Tricot in (Courbes et dimensions fractales, Springer, Berlin, 1995).
\end{abstract}

Keywords Hausdorff dimension - Sum of pulses · Box dimension

Mathematics Subject Classification (2000) $60 \mathrm{D} 05 \cdot 60 \mathrm{G} 99 \cdot 28 \mathrm{~A} 80$

\section{Introduction}

Let $(\Gamma, \mathcal{F}, \mathbb{P})$ be a probability space, we consider random functions of the type

$$
F(t)=\sum_{n=1}^{+\infty} a_{n} G\left(\lambda_{n}^{-1}\left(t-X_{n}\right)\right)
$$

E. de Amo · J. Fernández-Sánchez

University of Almeria, Almeria, Spain

E. de Amo

e-mail: edeamo@ual.es

I. Bhouri $(\bowtie)$

University of Monastir, Monastir, Tunisia

e-mail: imen.bhouri@fsm.rnu.tn 
where $\left(a_{n}\right),\left(\lambda_{n}\right)$ are two positive scalar sequences such that $\sum a_{n}$ is divergent, and $\left(\lambda_{n}\right)$ is non-increasing to 0 . The function $G: \mathbb{R} \rightarrow \mathbb{R}$ is the elementary bump (i.e an even continuous function supported on $[-1,1]$, decreasing on $[0,1]$ and satisfying $G(0)=1)$ and $X_{n}$ are continuous independent random variables uniformly distributed on a sufficiently large domain $\Omega$.

In the particular case $a_{n}=\frac{1}{n^{H}}, H \in(0,1)$ and $\lambda_{n}=\frac{1}{n}$, these functions have been introduced in [9] and [11] to generate measures associated to Poisson processes. In the same particular case and in higher dimension, the analysis of the fractal sums of pulses has been treated in [3] and [2]. The existence and regularity of functions defined by (1) have been studied in [1]. Notice that this kind of functions are important for the purpose of modeling strange phenomena which are known to exhibit multifractal behaviors. Such behaviors occur for instance in geophysics [5] when considering the spatial-temporal position and the intensity of seismic events, in telecommunications where the TCP Internet traffic is known to be multifractal [8], and also when studying financial time series [10]. This work was motivated by a question given in [13] about the Hausdorff dimension of the graph of functions defined by (1). In this paper, we investigate the Hausdorff dimension of their graphs which provides a measure of the irregularity of the process and gives a positive answer to the question of Tricot. In particular our result is an improvement of the result of [1] who gives only an upper bound of the upper box dimension of the graph of $F$.

The paper is organized as follows. In the next section we introduce some basic notions and properties. In Sect. 3 we state our main result giving the Hausdorff dimension of the pulse-sum functions. We prove Theorem 1 by using some potential theoretic methods for calculating the Hausdorff dimensions and some technical lemmas useful for our proof.

\section{Preliminaries}

Casually, we briefly recall some basic definitions and facts which will be used in subsequent developments.

Let $A$ be a subset of $\mathbb{R}^{2}$. The $s$-dimensional Hausdorff measure Hausdorff of $A$ is defined by

$$
\mathcal{H}^{s}(A)=\lim _{\varepsilon \rightarrow 0} \mathcal{H}_{\varepsilon}^{s}(A)
$$

where, for $\varepsilon>0$,

$$
\mathcal{H}_{\varepsilon}^{s}(A)=\inf \left\{\sum_{i=0}^{\infty}\left|E_{i}\right|^{s}: E \subset \bigcup_{i=0}^{\infty} E_{i} \text { and }\left|E_{i}\right| \leq \varepsilon\right\},
$$

with $|A|$ denoting the diameter of a set $A \in \mathbb{R}^{2}$. The Hausdorff dimension of $A$ is given by

$$
\operatorname{dim}(A)=\inf \left\{s: \mathcal{H}^{s}(A)=0\right\}=\sup \left\{s: \mathcal{H}^{s}(A)=\infty\right\}
$$

(see [4] and [12] for more details). When calculating the Hausdorff dimension of a set $A$, in general it is difficult to find a lower estimate of $\operatorname{dim}(A)$, and one approach is to relate Hausdorff dimension to certain energies. 
For $A \subset \mathbb{R}^{2}$, let

$$
\mathcal{M}(A)=\{\mu: \mu \text { is a finite Radon measure supported by } \mathrm{A}\} .
$$

For $\mu \in \mathcal{M}(A)$, we define the $s$-energy of $\mu$ by

$$
I_{s}(\mu)=\iint \frac{d \mu(x) d \mu(y)}{|x-y|^{s}} .
$$

Then

$$
\operatorname{dim}(A)=\sup \left\{s \geq 0: \exists \mu \in \mathcal{M}(A) \text { with } I_{s}(\mu)<\infty\right\}
$$

(see [4] and [12]). So, if we can construct a measure $\mu$ supported on $A$ with finite $s$-energy then $\operatorname{dim}(A) \geq s$. For the graph $\Gamma_{F} \subset \mathbb{R}^{2}$ of a continuous function $F:[0,1] \rightarrow \mathbb{R}$, there is a natural measure $\mu$ on $\Gamma_{F}$ as follows. If $\mathcal{L}^{1}$ denotes the Lebesgue measure on $[0,1]$,

$$
\mu(E)=\mathcal{L}^{1}\{t \in[0,1]:(t, F(t)) \in E\} \quad \text { for all } E \subset \mathbb{R}^{2} .
$$

If $x=(u, t) \in \mathbb{R}^{2}$, define $\|x\|_{2}=\left(u^{2}+t^{2}\right)^{1 / 2}$. We can rewrite (2) by

$$
I_{s}(\mu)=\iint_{[0,1]^{2}}\left((F(x)-F(y))^{2}+|x-y|^{2}\right)^{-s / 2} d x d y .
$$

\section{Results}

The existence and regularity of bumps sums functions defined by (1) have been studied in [1]. In particular Abid proved the following results. We denote by

$$
\Lambda_{j}=\left\{n: 2^{-j} \leq \lambda_{n}<2^{-(j-1)}\right\},
$$

and

$$
H=\liminf _{n \rightarrow \infty}\left(\inf _{n \in \Lambda_{j}} \frac{\log a_{n}}{\log \lambda_{n}}\right)
$$

Theorem (Ben Abid) 1 Assume that $\lambda_{n}=\frac{\alpha}{n}, \alpha>0$ and $G \in C^{1}(\mathbb{R})$. Then if $H \in(0,1]$ we have, almost surely, for every $\varepsilon \in(0, H), F \in C^{H-\varepsilon}(\mathbb{R})$.

Denote by $\Gamma_{F}:=\{(t, F(t)): t \in[0,1]\}$ the graph of the random function $F$. The Hölder estimates on $F$ immediately give an upper bound for the upper box-counting dimension $\overline{\operatorname{dim}}_{B} \Gamma_{F}$ of the graph (see [4]).

\section{Corollary (Ben Abid) 1 We have}

$$
\overline{\operatorname{dim}}_{B} \Gamma_{F} \leq 2-H, \quad \text { almost surely. }
$$

From now on $\lambda_{n}=\frac{\alpha}{n}$ with $\alpha>0$. Our main result is to calculate the Hausdorff dimension of the graph of $F$ which improves the result of Ben Abid and gives an answer to a question given by Tricot in [13]. 
Theorem 1 Assume that there exists a non-empty interval $I \subset[0,1]$ on which $G: I \rightarrow J$ is a $C^{1}$-diffeomorphism. Then we have

$$
\operatorname{dim} \Gamma_{F}=2-H, \quad \text { almost surely. }
$$

Since the Hausdorff dimension is less then its box dimension, due to Corollary 1, it is sufficient to prove that $\operatorname{dim} \Gamma_{F} \geq 2-H$, almost surely. The proof is based on the potential theoretic method to calculate the Hausdorff dimension of graphs of many functions, such as the fractional Brownian motion [7] or the random Weierstrass function [6] and those given in the particular case $a_{n}=\frac{1}{n^{H}}$ and $\lambda_{n}=\frac{1}{n}, H \in(0,1)$ in [2]. The potential theoretic ideas are developed in the following section.

In order to prove Theorem 1, we need some intermediate results. We use the following probability notations.

For each event $A \in \mathcal{F}$ with $\mathbb{P}(A)>0$ we write $\mathbb{P}^{A}$ for the probability conditional on $A$. We have $\mathbb{P}^{A}$ is absolutely continuous with respect to $\mathbb{P}$ with density $\frac{d \mathbb{P}^{A}}{d \mathbb{P}}=\frac{1}{\mathbb{P}(A)} \chi_{A}$. We denote by $\mathbb{E}^{A}$ the expectation with respect to $\mathbb{P}^{A}$ to get for all random variables $Y$, $\mathbb{E}^{A}(Y)=\frac{1}{\mathbb{P}(A)} \mathbb{E}\left(Y \chi_{A}\right)$. Further, we write $\mathbb{P}_{Y}$ for the law of $Y$ as a random variable on $(\Gamma, \mathcal{F}, \mathbb{P})$.

For $x, y \in[0,1]$ we define

$$
Z=F(x)-F(y)=\sum_{n=1}^{\infty} Z_{n}
$$

where

$$
Z_{n}=a_{n}\left(G\left(\lambda_{n}^{-1}\left(x-X_{n}\right)\right)-G\left(\lambda_{n}^{-1}\left(y-X_{n}\right)\right)\right) .
$$

For this fixed $x$, we write $A_{n}$ for the event $\left(x \in C_{n}^{\prime}\right)$ where

$$
C_{n}^{\prime}=\left\{t \in \mathbb{R}:\left|t-X_{n}\right| \lambda_{n}^{-1} \in I\right\} .
$$

The results of the following lemmas are similar to Lemma 3.1, Corollary 3.2 and Corollary 3.3 established in [2].

Lemma 1 Let $x, y \in[0,1]$ be fixed. For all $p \geq 1$ such that $|x-y|>2 \lambda_{p}$, the random variable $Z_{p}$ has a density conditional on $A_{p}$ given by

$$
f_{p}(z)=\frac{\lambda_{p}}{a_{p} \mathbb{P}\left(A_{p}\right)}\left|h^{\prime}\left(\frac{z}{a_{p}}\right)\right| \chi_{J}\left(\frac{z}{a_{p}}\right) \quad \text { for all } z \in \mathbb{R},
$$

where $h: J \rightarrow I$ is the inverse of $G$.

Now denote by $S_{p}=\sum_{n \neq p} Z_{n}$ so that $Z=S_{p}+Z_{p}$. We condition on $S_{p}$ and we regard $Z$ as random variable on $\left(\Gamma, \mathcal{F}, \mathbb{P}^{\mathbb{A}_{p}}\right)$.

Lemma 2 Let $x, y \in[0,1]$ and $p \geq 1$ such that $|x-y|>2 \lambda_{p}$. Then $Z$ has a density conditional on $S_{p}$ given by

$$
f_{z}^{S_{p}=s}(z)=f_{p}(z-s) \quad \text { for all } z \in \mathbb{R}
$$

where $f_{p}$ is as in Lemma 1. 
Lemma $3 \forall n>m \geq 1, \forall x, y \in[0,1]$ such that $|x-y|>2 \lambda_{m}$ and $r>0$, we have

$$
\mathbb{P}\left((|F(x)-F(y)|<r) \cap\left(A_{m} \cup \cdots \cup A_{n}\right)\right) \leq C \frac{r}{a_{n}}
$$

for some $C>0$.

Lemma 4 Let $s>1$. For $1 \leq m<n$, let $V=C_{m} \cup \cdots \cup C_{n}$. For $x, y \in[0,1]$ such that $|x-y|>2 \lambda_{m}$, we have

$$
\mathbb{E}\left(\left((F(x)-F(y))^{2}+|x-y|^{2}\right)^{-s / 2} \chi_{(x \in V)}\right) \leq C|x-y|^{1-s} \frac{1}{a_{n}}
$$

for some $C>0$.

Proof of Lemma 4 Denote $h=|x-y|$, for $r>0$ due to Lemma 3 we have

$$
p(r):=\mathbb{P}((|Z|<r) \cap(x \in V)) \leq C \frac{r}{a_{n}} .
$$

So,

$$
\begin{aligned}
\mathbb{E}^{(x \in V)}\left(\left(|Z|^{2}+h^{2}\right)^{-s / 2}\right) & =\int_{0}^{\infty}\left(r^{2}+h^{2}\right)^{-s / 2} d\left(\mathbb{P}^{(x \in V)}(|Z|<r)\right) \\
& =\frac{1}{\mathbb{P}(x \in V)} \int_{0}^{\infty}\left(r^{2}+h^{2}\right)^{-s / 2} d p(r) .
\end{aligned}
$$

As a consequence,

$$
\mathbb{E}^{(x \in V)}\left(\left(|Z|^{2}+h^{2}\right)^{-s / 2}\right) \chi_{(x \in V)}=\int_{0}^{\infty}\left(r^{2}+h^{2}\right)^{-s / 2} d p(r) .
$$

Integrating by parts we get,

$$
\begin{aligned}
\int_{0}^{\infty}\left(r^{2}+h^{2}\right)^{-s / 2} d p(r) & \leq \int_{0}^{h} h^{-s} d p(r)+\int_{h}^{\infty} r^{-s} d p(r) \\
& \leq h^{-s} p(h)+\left[r^{-s} p(r)\right]_{r=h}^{\infty}+s \int_{h}^{\infty} r^{-s-1} p(r) d r \\
& \leq C h^{-s} \frac{h}{a_{n}}+C s \int_{h}^{\infty} r^{-s-1} \frac{r}{a_{n}} d r \leq \frac{C}{a_{n}} h^{1-s}
\end{aligned}
$$

and (6) yields.

Next we want to prove that for given $x, y$, the quantity $|F(x)-F(y)|$ is of high probability of being suitably large, for $x$ in a large random subset of $[0,1]$.

Remark 1 Recall that $H$ is defined by (4) so for all $\varepsilon \in(0, H)$, there exists $k_{\varepsilon} \geq 1$ such that for all $k \geq k_{\varepsilon}$, for all $n \in \Lambda_{k}, a_{n} \geq 2^{-k H(1+\varepsilon / 2)}$.

The following result is a straightforward consequence of Lemma 4 by considering the random set $V_{k}=C_{n_{k^{2}}}^{\prime} \cup \cdots \cup C_{n_{(k+1)^{2}}}^{\prime}$ with $n_{j} \in \Lambda_{j}$ for $(k+1)^{2}-1 \leq j \leq k^{2}$. 
Corollary 1 Let $s>1, \varepsilon>0$ and $x, y \in[0,1]$ such that $|x-y|<2 \lambda_{k_{\varepsilon}-1}$. Let $k \geq 1$ be the unique integer satisfying $2 \lambda_{k}<|x-y| \leq 2 \lambda_{k-1}$. Then

$$
\mathbb{E}\left(\left((F(x)-F(y))^{2}+|x-y|^{2}\right)^{-s / 2} \chi_{\left(x \in V_{k}\right)}\right) \leq C|x-y|^{1-s-H(1+\varepsilon / 2)}
$$

for some $C>0$.

Further we will estimate the measure of $V_{k}$.

Lemma 5 There exists a constant $\delta>0$ such that for all $1 \leq m<n$, we have

$$
\mathbb{E}\left(\mathcal{L}^{1}\left([0,1] \backslash \bigcup_{p=m}^{n} C_{p}\right)\right) \leq\left(\frac{m}{n}\right)^{\delta} .
$$

For the proof of this lemma see Lemma 3.6 in [2].

\subsection{Proof of Theorem 1}

Let $1<s<2-H$. Choose $\varepsilon>0$ such that $(1+\varepsilon / 2) H<2-s<1$ with $k_{\varepsilon} \geq 1$ the associated integer. Fix $k_{0} \geq k_{\varepsilon}$, we define $W=[0,1] \cap\left(\bigcap_{k=k_{0}}^{\infty} V_{k}\right)$. The proof of Theorem 1 splits in two steps. Denote by $\mathcal{L}^{1}{ }_{W}$ the restriction of Lebesgue measure to $W$ and $R_{k}=\left\{(x, y) \in[0,1] \times[0,1]: 2 \lambda_{k}<|x-y| \leq 2 \lambda_{k-1}\right\}$.

Step 1. From the definition of $W$ and due to Corollary 1 we have

$$
\begin{aligned}
& \mathbb{E}\left(\iint_{\left\{x, y \in[0,1]:|x-y| \leq 2 \lambda_{k_{0}}\right\}}\left((F(x)-F(y))^{2}+|x-y|^{2}\right)^{-s / 2} d \mathcal{L}^{1}{ }_{W}(x) d \mathcal{L}^{1}{ }_{W}(y)\right) \\
& \leq \mathbb{E}\left(\iint_{\left\{x \in W, y \in[0,1]:|x-y| \leq 2 \lambda_{k_{0}}\right\}}\left((F(x)-F(y))^{2}+|x-y|^{2}\right)^{-s / 2} d x d y\right) \\
& \leq \mathbb{E}\left(\sum_{k=k_{0}}^{\infty} \iint_{R_{k} \cap W \times[0,1]}\left((F(x)-F(y))^{2}+|x-y|^{2}\right)^{-s / 2} d x d y\right) \\
& \leq \mathbb{E}\left(\sum_{k=k_{0}}^{\infty} \iint_{R_{k}}\left((F(x)-F(y))^{2}+|x-y|^{2}\right)^{-s / 2} \chi_{\left(x \in V_{k}\right)} d x d y\right) \\
& \leq \sum_{k=k_{0}}^{\infty}\left(\iint_{R_{k}} \mathbb{E}\left(\left((F(x)-F(y))^{2}+|x-y|^{2}\right)^{-s / 2} \chi_{\left(x \in V_{k}\right)}\right) d x d y\right) \\
& \leq C \sum_{k=k_{0}}^{\infty}\left(\iint_{R_{k}}|x-y|^{1-s-H(1+\varepsilon / 2)} d x d y\right) \\
& \leq C \int_{\left\{x \in W, y \in[0,1]:|x-y| \leq 2 \lambda_{k_{0}}\right\}}|x-y|^{1-s-H(1+\varepsilon / 2)} d x d y,
\end{aligned}
$$


since $1-s-H(1+\varepsilon)>-1$, this last integral converges, therefore the integral

$$
\iint_{\left\{x, y \in[0,1]:|x-y| \leq 2 \lambda_{k_{0}}\right\}}\left((F(x)-F(y))^{2}+|x-y|^{2}\right)^{-s / 2} d \mathcal{L}^{1}{ }_{W}(x) d \mathcal{L}^{1}{ }_{W}(y)
$$

is finite almost surely and so

$$
\iint_{[0,1] \times[0,1]}\left((F(x)-F(y))^{2}+|x-y|^{2}\right)^{-s / 2} d \mathcal{L}^{1}{ }_{W}(x) d \mathcal{L}^{1}{ }_{W}(y)<\infty
$$

almost surely.

Step 2. Let $\mu_{W}$ be the finite Borel measure on $\mathbb{R}^{2}$ defined by $\mu_{W}(E)=\mathcal{L}^{1}\{t \in W$ : $(t, F(t)) \in E\}$ for all $E \subset \mathbb{R}^{2}$. Notice that $\mu_{W}$ is supported on $\Gamma_{F}$ and of finite $s$-energy. Hence, to conclude that $\operatorname{dim} \Gamma_{F} \geq s$ it is sufficient to prove that $\mu_{W}$ is positive which is equivalent to show that $\mathcal{L}^{1}(W)>0$.

We have $[0,1] \backslash W=\bigcup_{k=k_{0}}^{\infty}\left([0,1] \backslash \bigcup_{p=n_{k^{2}}}^{n} C_{k}\right)$ so by Lemma 5

$$
\mathbb{E}\left(\mathcal{L}^{1}([0,1] \backslash W)\right) \leq \sum_{k=k_{0}}^{\infty}\left(\frac{n_{k^{2}}}{n_{(k+1)^{2}}-1}\right)^{\delta}
$$

Since $n_{k^{2}} \in \Lambda_{k^{2}}$ then $\alpha 2^{k^{2}-1} \leq n_{k^{2}}<\alpha 2^{k^{2}}$.

Hence

$$
\mathbb{E}\left(\mathcal{L}^{1}([0,1] \backslash W)\right) \leq \sum_{k=k_{0}}^{\infty} 2^{-2 k \delta}=\frac{2^{-2 k_{0} \delta}}{1-2^{-2 \delta}} .
$$

Using Markov's inequality we have,

$$
\mathbb{P}\left(\mathcal{L}^{1}(W)<1 / 2\right)=\mathbb{P}\left(\mathcal{L}^{1}([0,1] \backslash W) \geq 1 / 2\right) \leq 2 \frac{2^{-2 k_{0} \delta}}{1-2^{-2 \delta}} .
$$

Let $0<\eta<1$ and choose $k_{0}$ large enough such that $\frac{2^{1-2 k_{0} \delta}}{1-2^{-2 \delta}}<\eta$, so $\mathcal{L}^{1}(W) \geq 1 / 2$ with probability greater than $1-\eta$. From the previous two steps we conclude that $\operatorname{dim} \Gamma_{F} \geq s$ with probability at least $1-\eta$. The arbitrariness on $s$ and $\eta$ implies that $\operatorname{dim} \Gamma_{F} \geq 2-H$ almost surely.

Open Access This article is distributed under the terms of the Creative Commons Attribution Noncommercial License which permits any noncommercial use, distribution, and reproduction in any medium, provided the original author(s) and source are credited.

\section{References}

1. Ben Abid, M.: Existence et régularité holdérienne des fonctions de bosses. Colloq. Math. 116(2), 217225 (2009)

2. Demichel, Y., Falconer, K.J.: The Hausdorff dimension of pulse-sum graphs. Math. Proc. Camb. Phil. Soc. 143, 145-155 (2007)

3. Demichel, Y., Tricot, C.: Analysis of the fractal sum of pulses. Math. Proc. Camb. Phil. Soc. 141, 355370 (2006)

4. Falconer, K.J.: Fractal Geometry. Mathematical Foundations and Applications. Wiley, New York (1990)

5. Harte, D.: Multifractals: Theory and Applications. Chapman \& Hall, London (2001) 
6. Hunt, B.R.: The Hausdorff dimension of graphs of Weirstrass functions. Proc. Am. Math. Soc. 126, 791-800 (1998)

7. Kahane, J.P.: Some Random Series of Functions. Cambridge University Press, Cambridge (1985)

8. Lévy Véhel, J., Riedi, R.H.: TCP traffic is multifractal: a numerical study. INRIA Research Report, RR-3129 (1997)

9. Mandelbrot, B.: Introduction to fractal sums of pulses. In: Lévy Process and Related Phenomena in Physics. Lect. Notes Physics, vol. 450, pp. 110-123. Springer, Berlin (1995)

10. Mandelbrot, B.: Fractals and Scaling in Finance (Discontinuity, Concentration, Risk). Springer, Berlin (1997)

11. Mandelbrot, B., Barral, J.: Multifractal products of cylinder pulses. Probab. Theory Relat. Fields 124, 409-430 (2002)

12. Mattila, P.: Geometry of Sets and Measures in Euclidean Spaces, Fractals and Rectifiability. Cambridges Studies in Advanced Mathematics, vol. 44. Cambridge University Press, Cambridge (1995)

13. Tricot, C.: Courbes et Dimensions Fractales. Springer, Berlin (1995) 